\title{
Notes on the Variety of Ternary Algebras
}

\author{
Aldo V. Figallo', Claudia M. Gomes' ${ }^{1}$, Lucía S. Sarmiento², Mario E. Videla² \\ ${ }^{1}$ Instituto de Ciencias Básicas, Universidad Nacional de San Juan, San Juan, Argentina \\ ${ }^{2}$ Departamento de Matemática, Universidad Nacional de San Juan, San Juan, Argentina \\ Email: avfigallo@gmail.com, gomescostela@gmail.com, lussarmiento@gmail.com, maredvid@gmail.com
}

Received 5 July 2014; revised 3 August 2014; accepted 12 August 2014

Copyright @ 2014 by authors and Scientific Research Publishing Inc.

This work is licensed under the Creative Commons Attribution International License (CC BY).

http://creativecommons.org/licenses/by/4.0/

(c) (i) 0 pen Access

\section{Abstract}

In this work we review the class $\mathcal{T}$ of ternary algebras introduced by J. A. Brzozowski and C. J. Serger in [1]. We determine properties of the congruence lattice of a ternary algebra $A$. The most important result refers to the construction of the free ternary algebra on a poset. In particular, we describe the poset of the join irreducible elements of the free ternary algebra with two free generators.

\section{Keywords}

De Morgan Algebra, Free Algebra, Congruence, Variety

\section{Introduction and Preliminaries}

Different algebras began to be used in the thirties as a tool for the analysis and design of digital circuits. One of the pioneers in this field was C. E. Shannon, who in 1938 applied Boolean algebras in his work " A Symbolic Analysis of Change and Retransmission circuits". Even though technology has changed substantially, these algebras still play a very important role in this field. In contrast, the use of ternary algebras has been much more limited, and the first applications of trivaluada logic to switching circuits were made by Goto in 1948. He worked with the algebra $A=\langle\{0,1 / 2,1\},+, \cdot,-, 0,1\rangle$, of type $(2,2,1,0,0)$, where $a+b=\max \{a, b\}$,

$a \cdot b=\min \{a, b\}, \bar{a}=1-a$. He interpreted $1 / 2$ as an indefinite value, and used it to represent a transition state between 0 and 1 . In addition, by means of the resolution of trivalued logic equations, he managed to derive the necessary conditions to prevent hazardous behavior in sequential relay contact networks. In 1959, Muller worked with this same ternary algebra, and applied it to the study of the transient phenomena in switching circuits, and observed, among other things, that it satisfies Kleene's law: $(K)(a \cdot \bar{a})+(b+\bar{b})=b+\bar{b}$.

In 1964, Yoeli and Rinon applied them to the study of static hazards in combinational switching circuits. In 1965, Eichelberger used them in the analysis of hazard phenomena in combinational and sequential switching circuits. 
In 1983, Mukaidono studied a special class of ternary functions, the regular ones, and indicated a set of axioms to define a ternary algebra, calling the resulting algebra Kleene algebra with a centre. However, it was not until 1995 that Brozozowski and Serger presented them as a De Morgan algebra with an additional constant, $e$, which satisfies these identities:

1) $\bar{e}=e$,

2) $(a+\bar{a})+e=a+\bar{a}$.

The same year Negulescu introduced the process spaces, a formalism which allows the modelling of the behaviour of interacting systems.

In 1996, Brozozowski, Lou and Negulescu considered certain subalgebras of the process spaces, i.e. the subset-pair algebras, and showed that each finite ternary algebra is isomorphic to a subset-pair algebra. In 1997, Esik showed that this property is valid for any ternary algebra and that, in general, each ternary algebra is isomorphic to an algebra of ternary functions, this result being analogous to Caley's representation for Boolean algebras.

This class of algebras is applied in the detection of hazards in the combinational circuits, in the modelling of interacting systems by means of process spaces and in the analysis of asynchronous sequential circuits by means of Eichelberger's method of ternary simulation, among other applications.

This article is organized as follows. Firstly, we summarize the main definitions and necessary results for a better comprehension of the article. In Section 2, we characterize the subdirectly irreducible algebras, examine the congruence lattice of a ternary algebra and establish properties of the variety $\mathcal{T}$. Finally, in Section 4 , we indicate a construction of the free ternary algebra on a poset, and apply it to the free ternary algebra on one free generator and describe the poset of the join irreducible elements of the free ternary algebra on two free generators.

Definition 1.1 A ternary algebra is an algebra $\mathbf{L}=\langle L,+, \cdot, \sim, 0, e, 1\rangle$ of type $(2,2,1,0,0,0)$ which satisfies these conditions:

(T1) $\langle L,+, \cdot, \sim, 0,1\rangle$ is a De Morgan algebra,

(T2) $(a+\sim a)+e=a+\sim a$ for all $a \in L$,

(T3) $\sim e=e$.

This class of algebras forms a variety which will be denoted by $\mathcal{T}$.

The following properties are satisfied on $\mathcal{T}$ :

(T4) $(a \cdot \sim a) \cdot e=a \cdot \sim a$ for all $a \in L$.

(T5) $(a+\sim a)+(b \cdot \sim b)=a+\sim a$ for all $a, b \in L$.

(T6) In each ternary algebra the only element that satisfies the condition $\sim x=x$ is $e$.

(T7) Every finite ternary algebra has an odd number of elements.

(T8) There exist, up to isomorphism, one ternary algebra with five elements, two with seven, and four with nine.

(T9) For each odd natural number $n \geq 3$, there exists at least one ternary algebra with $n$ elements.

Clearly, (T4) is the dual property of (T2) while (T5) is equivalent to (T2).

Two well known examples of ternary algebras, of significant importance for the rest of this work, are:

\section{Example 1.1}

1) $T_{0}$ is the ternary algebra $\langle\{0,1 / 2,1\},+, \cdot,, 0,1 / 2,1\rangle$, where $a+b=\max \{a, b\}, a \cdot b=\min \{a, b\}, \sim 0=1$, $\sim 1=0$ and $\sim 1 / 2=1 / 2$.

2) For each odd integer $n>3, T_{n}$ is the ternary algebra $\langle\{0,1,2, \cdots, n-1\},+, \cdot, \sim, 0,(n-1) / 2, n-1\rangle$, where $a+b=\max \{a, b\}, a \cdot b=\min \{a, b\} \quad y \sim a=(n-1)-a$.

\section{The Congruence Lattice and Subdirectly Irreducible Algebras}

In order to characterize the subdirectly irreducible ternary algebras we first observe that the ternary algebras arises from Kleene algebras endowed with a nullary operation.

Definition 2.1 A Kleene algebra $\langle L,+, \cdot \sim, 0,1\rangle$ is a De Morgan algebra which satisfies the property (K) $x \cdot \sim x \leqslant y+\sim y$ for every $x, y \in L$.

Definition 2.2 A Kleene algebra $\langle L,+, \cdot, \sim, 0,1\rangle$ is centered if there exists an element $c \in L$ such that $\sim c=c, c$ is said the centre of $L$.

We represent this class of algebras by $\mathcal{K}_{C}$. 
Remark 2.1 From Definitions 2.1 y 2.2 we have that:

1) If $\langle L,+, \cdot \sim, 0, e, 1\rangle \in \mathcal{T}$, then the reduct $\hat{L}=\langle L,+, \cdot, \sim, 0,1\rangle \in \mathcal{K}_{C}$, with centre in $e$.

2) If $\langle L,+, \cdot, \sim, 0,1\rangle \in \mathcal{K}_{C}$ with centre in $e$, entonces $\langle L,+, \cdot, \sim, 0, e, 1\rangle \in \mathcal{T}$.

Well known results from Kleene algebra theory allow us to state the following:

Theorem 2.1 The only subdirectly irreducible centered Kleene algebra is the three-elements chain.

As a direct consequence of Theorem 2.1, we can establish

Corollary 2.1 The only subdirectly irreducible ternary algebra is $T_{0}=\{0, e, 1\}$.

The preceding results and some from universal algebra allow us to ensure that:

Theorem 2.2

1) The simple algebras in this variety coincide with the subdirectly irreducible.

2) Variety $\mathcal{T}$ is semisimple, residually finite and locally finite.

Besides, with respect to the directly indecomposable algebras we can state that:

Theorem 2.3 In $\mathcal{T}$ there exist directly indecomposable algebras which are not subdirectly irreducible.

Proof. Any ternary algebra $T_{p}$ with $p$ a prime number greater than or equal to 3 is directly indecomposable but not subdirectly irreducible.

From the above results we can conclude that:

Corollary 2.2 Variety $\mathcal{T}$ is not directly representable.

\section{Lattice $\operatorname{Con}_{\mathcal{T}}(L)$}

Now we will consider the congruence lattice of a ternary algebra $L$, $\operatorname{Con}_{\mathcal{T}}(L)$, and will establish some of its most relevant properties.

Theorem 2.4 Variety $\mathcal{T}$ is not congruence-permutable.

Proof. In the ternary algebra $T_{5}$, the congruences $\theta_{1}, \theta_{2} \in \operatorname{Con}_{\mathcal{T}}\left(T_{5}\right)$ associated to partitions $\mathcal{P}_{1}=\{\{0,1\},\{2\},\{3,4\}\}$ and $\mathcal{P}_{2}=\{\{0\},\{1,2,3\},\{4\}\}$ of the set $\{0,1,2,3,4\}$ respectively, satisfy that $\theta_{1} \circ \theta_{2} \neq \theta_{2} \circ \theta_{1}$,

Theorem 2.5 Variety $\mathcal{T}$ is not congruence-uniform.

Proof. It is sufficient to consider the equivalence classes $[1]_{\theta_{1}}$ and $[2]_{\theta_{1}}$ in the congruence $\theta_{1} \in \operatorname{Con}_{\mathcal{T}}\left(T_{5}\right)$ considered in the proof of the theorem 2.4.

Theorem 2.6 Variety $\mathcal{T}$ is not congruence-regular.

Proof. In the ternary algebra $T_{7}$, it is sufficient to consider the congruences $\theta_{1}, \theta_{2} \in \operatorname{Con}_{\mathcal{T}}\left(T_{7}\right)$ associated to partitions $\mathcal{P}_{1}=\{\{0,1\},\{2\},\{3\},\{4\},\{5,6\}\}$ and $\mathcal{P}_{2}=\{\{0,1\},\{2,3,4\},\{5,6\}\}$ of the set $\{0,1,2,3,4,5,6\}$, respectively.

As a direct consequence of Theorems 2.4 y 2.5, we can formulate:

Corollary 2.3 Variety $\mathcal{T}$ is neither arithmetic nor discriminatory.

The equations that define the principal congruences in the De Morgan algebras obtained by H. P. Sankappanavar in [2] allows the following:

Theorem 2.7 Variety $\mathcal{T}$ has equationally definable principal congruences.

From the results obtained by A. Day in 1971 [3], and for W. J. Blok y D. Pigozzi in 1982 [4], we can conclude that:

\section{Theorem 2.8}

1) Variety $\mathcal{T}$ is congruence-distributive, it has the congruence extension property and the principal congruence extension property.

2) $\theta_{B}(a, b)=\theta_{A}(a, b) \mid B$ for all $A, B \in \mathcal{T}$, being $B$ a subalgebra of $A$ and $a, b \in B$.

In addition, taking into account the characterization given by P. Kholer y D. Pigozzi in 1980 [5], we have that:

Theorem 2.9 For all $L \in \mathcal{T},\left\langle C_{P}(L), \vee, I d_{L}\right\rangle$ is a $\vee$-semilattice with dual relative pseudo-complement, where $C_{P}(L)$ is the set of the finitely generated congruences of the algebra $L$.

\section{Free Ternary Algebras over an Poset}

In 1945, R. Dilworth [6] introduced the notion of free lattice over a poset. Later on, this subject was studied by many authors and in different classes of algebras. In this section we indicate a construction of the free ternary algebra over a poset, a result that generalizes those obtained by R. Balbes over free ternary algebras [7].

Let $\mathcal{K}$ be a variety of similar algebras which have an underlying ordered structure definable by a set of equ- 
ations $p_{i}(x, y)=q_{i}(x, y), \quad 1 \leq i \leq n$, which are in terms of the algebra operations. The notion of free algebra over an poset $I$ on $\mathcal{K}$ can be defined as follows:

Definition 3.1 A $\mathcal{K}$-algebra $L_{\mathcal{K}}(I)$ is free over $I$ if it satisfies the conditions that follow:

(L1) there exists an order-embedding $g: I \rightarrow L_{\mathcal{K}}(I)$ such that $L_{\mathcal{K}}(I)=[g(I)]_{\mathcal{K}}$ where $[X]_{\mathcal{K}}$ is the $\mathcal{K}$ - subalgebra of $L_{\mathcal{K}}(I)$ generated by $X$,

(L2) for each $\mathcal{K}$-algebra $A$ and each increasing function $f: I \rightarrow A$, there exists a homomorphism $h: L_{\mathcal{K}}(I) \rightarrow A$ such that $h \circ g=f$.

Remark 3.1 It is clear that:

1) If the free algebra over an poset $I$ exists, it is unique up to isomorphism.

2) The homomorphism $h$ is unique.

3) If $I$ is an poset of cardinality $n, n>0$, and the order over $I$ is the identity, then $L_{\mathcal{K}}(I)$ is the free algebra with a set of $n$ free generators.

4) In variety $\mathcal{T}$ each algebra has a underlying ordered structure defined by the equation $x \leqslant y$ if, and only if, $x=x \cdot y$.

In what follows, we determine the structure of $L_{\mathcal{T}}(I)$.

\subsection{Construction of the Free Ternary Algebra over a Poset I}

A. Figallo Jr. and A. Ziliani in [8] construct the free algebra over a poset in finitely generated varieties; by using a similar argument, we indicate a construction of the free ternary algebra over a poset.

We consider a poset $I$, the set $E$ of the increasing functions of $I$ in the ternary algebra $T_{0}$ and the function $g: I \rightarrow T_{0}^{E}$ defined by $g(i)=G_{i}$ where $G_{i}: E \rightarrow T_{0}$ is such that $G_{i}(f)=f(i)$ for each $f \in E$ and for each $i \in I$.

Theorem 3.1 The free ternary algebra $L_{\mathcal{T}}(I)$ over the poset $I$ is $[G]_{\mathcal{T}}$ where $G=\left\{G_{i}: i \in I\right\} \subseteq T_{0}^{E}$.

Proof.

(L1) It is clear that if $i, j \in I$ are such that $i \leqslant j$, then $G_{i} \leqslant G_{j}$.

Conversely, let us suppose that $i \not j j$, the function $f: I \rightarrow T_{0}$ defined by

$$
f(x)= \begin{cases}e & \text { if } x=i, \\ 0 & \text { if } x<i \quad 0 x \leqslant j, \\ 1 & \text { otherwise }\end{cases}
$$

is an increasing function such that $G_{i}(f) \nless G_{j}(f)$. From what has been stated, we conclude that $i \leqslant j$ on $I$ if, and only if, $G_{i} \leqslant G_{j}$ on $G$, from which it turns out that $g: I \rightarrow g(I)$ is an order isomorphism, such that $[g(I)]_{\mathcal{T}}=[G]_{\mathcal{T}}=L_{\mathcal{T}}(I)$. Therefore, (L1) holds.

(L2) Let $L$ be a ternary algebra and $f: I \rightarrow L$ an increasing function, by Corollary 2.1, $L$ is isomorphic to a subalgebra of $T_{0}^{X}$ being $X$ an arbitrary set, then there exists a subalgebra $S$ of $T_{0}^{X}$ and an isomorphism $\varphi: L \rightarrow S$. We claim that there is a homomorphism $h:[G]_{\mathcal{T}} \rightarrow T_{0}^{X}$ such that $h \circ g=\varphi \circ f$. Indeed, for each $x \in X$ let us consider the function $t_{x}: I \rightarrow T_{0}$ defined by $\left(t_{x}\right)(i)=((\varphi \circ f)(i))(x)$. It is easily shown that $t_{x} \in E$ for each $x \in X$, therefore $t: X \rightarrow E$ defined by $t(x)=t_{x}$ is function. From what has been stated it follows that the function $h:[G]_{\mathcal{T}} \rightarrow T_{0}^{X}$ defined by $h(F)=F \circ t$ is a homomorphism such that $h \circ g=\varphi \circ f$. In addition, as $h$ is a homomorphism and $S^{\prime}=\left\{F \in T_{0}^{X}: h(F) \in S\right\}$ is a ternary subalgebra of $T_{0}^{X}$ which contains $G$, it turns out that $h\left([G]_{\mathcal{T}}\right) \subseteq S$. Therefore, (L2) holds.

\subsection{Free Ternary Algebra on One Free Generator}

If $L$ is a ternary algebra and $S \subseteq L$, we denote by $[S]_{\mathcal{T}}$ the ternary algebra generated by the set $S$ and by $\mathcal{T}[S]$ the free ternary algebra with $S$ as the set of free generators, and we set $\sim S=\{\sim s: s \in S\}$ and $\bar{S}=S \cup\{e\}$.

In [7], R. Balbes proves that $[X]_{\mathcal{T}}=\left\{\sum_{i=1}^{n} \prod S_{i}: S_{i} \subseteq \bar{X} \cup \sim \bar{X}\right.$ and $\left.n \geq 0\right\}$, we apply this result and those obtained before in order to get the free ternary algebra on one free generator. 
Let $I=\left\{i_{0}\right\}$ and $E=\left\{f: I \rightarrow T_{0}: f\right.$ is increasing $\}=\left\{f_{1}, f_{2}, f_{3}\right\}$ where $f_{1}, f_{2}, f_{3}$ are defined as follows $f_{1}\left(i_{0}\right)=0, \quad f_{2}\left(i_{0}\right)=e$ y $f_{3}\left(i_{0}\right)=1$

Let $g: I \rightarrow T_{0}^{E}$ defined by $g\left(i_{0}\right)=G_{i_{0}}$ where $G_{i_{0}}: E \rightarrow T_{0}$ such that $G_{i_{0}}\left(f_{1}\right)=0, \quad G_{i_{0}}\left(f_{2}\right)=e$ y $G_{i_{0}}\left(f_{3}\right)=1$. Some of the elements of $T_{0}^{E}$ are specified in the Table 1 .

Then, the free ternary algebra on one free generator is:

$$
L_{\mathcal{T}}\left(\left\{i_{0}\right\}\right)=[G]_{\mathcal{T}}=\left\{\sum_{i=1}^{n} \prod S_{i}: S_{i} \subseteq \bar{G} \cup \sim \bar{G} \text { y } n \geq 0\right\}
$$

where $G=\left\{G_{i_{0}}\right\}$. Then, $L_{\mathcal{T}}\left(\left\{i_{0}\right\}\right)=\left\{G_{i_{0}}, F_{e}, F_{0}, F_{1}, F_{4}, F_{8}, F_{7}, F_{9}, F_{11}, F_{10}, F_{12}\right\}=(3 \times 3)_{01}$ which coincides with the result obtained by R. Balbes en [7].

The algebra $L_{\mathcal{T}}\left(\left\{i_{0}\right\}\right)$ is that indicated in Figure 1.

\subsection{The Poset of the Join Irreducible Elements of the Free Ternary Algebra on Two Free Generators}

In [7] R. Balbes proves that the poset of the join irreducible elements of the free ternary algebra $\mathcal{T}[X]$ with a finite set of free generators $X$ is isomorphic with $I P=I P_{1}(X) \cup I P_{2}(X)$, being

$$
I P_{1}(X)=\{S \subseteq \bar{X} \cup \sim \bar{X}: S \cap \sim S=\varnothing\} \text { and } I P_{2}(X)=\{S \subseteq \bar{X} \cup \sim \bar{X}: \bar{X} \subseteq S \cup \sim S\},
$$

ordered by the relation $T \leqslant S$ if, and only if, $S \subseteq T$.

We apply this result and those obtained above in order to determine the poset of the join irreducible elements of a free ternary algebra on two free generators. We consider the set $I=\left\{i_{0}, j_{0}\right\}$ ordered by the identity relation, $E=\left\{f: I \rightarrow T_{0}: f\right.$ is increasing $\}=\left\{f_{1}, f_{2}, f_{3}, f_{4}, f_{5}, f_{6}, f_{7}, f_{8}, f_{9}\right\}$ where each of the functions are defined in the Table 2, and $g: I \rightarrow T_{0}^{E}$ defined by

Table 1. Elements of $T_{0}^{E}$.

\begin{tabular}{ccccccccccccccccc}
\hline$E$ & $G_{i 0}$ & $F_{0}$ & $F_{e}$ & $F_{1}$ & $F_{2}$ & $F_{3}$ & $F_{4}$ & $F_{5}$ & $F_{6}$ & $F_{7}$ & $F_{8}$ & $F_{9}$ & $F_{10}$ & $F_{11}$ & $F_{12}$ \\
\hline$f_{1}$ & 0 & 0 & $e$ & 1 & 0 & 0 & 0 & $\mathrm{e}$ & 1 & 1 & 0 & $e$ & 1 & $e$ & 1 \\
$f_{2}$ & $e$ & 0 & $e$ & 1 & 0 & 0 & $e$ & 0 & 0 & $e$ & $e$ & $e$ & $e$ & $e$ & $e$ \\
$f_{3}$ & 1 & 0 & $e$ & 1 & $e$ & 1 & 0 & 0 & 0 & 0 & $e$ & 0 & $e$ & 1 & 1 \\
\hline
\end{tabular}

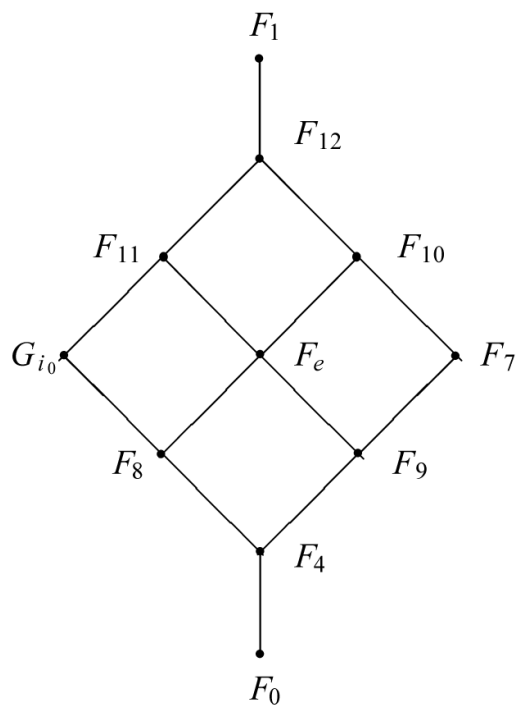

Figure 1. The free ternary algebra on one free generator. 


$$
g(x)=\left\{\begin{array}{lll}
G_{i_{0}} & \text { si } & x=i_{0} \\
G_{j_{0}} & \text { si } & x=j_{0}
\end{array}\right.
$$

where $G_{i}: E \rightarrow T_{0}$ such that $G_{i}(f)=f(i)$ for each $i \in I$.

In the Table 3, we specify some of the elements of $T_{0}^{E}$.

Table 2. The elements of $E$.

\begin{tabular}{ccccccccccc}
\hline$I$ & $f_{1}$ & $f_{2}$ & $f_{3}$ & $f_{4}$ & $f_{5}$ & $f_{6}$ & $f_{7}$ & $f_{8}$ & $f_{9}$ \\
\hline$i_{0}$ & 0 & $e$ & 1 & 0 & 0 & $e$ & $e$ & 1 & 1 \\
$j_{0}$ & 0 & $e$ & 1 & $e$ & 1 & 0 & 1 & 0 & $e$
\end{tabular}

Table 3. Elements of $T_{0}^{E}$.

\begin{tabular}{ccccccccccccccccc}
\hline $\mathrm{E}$ & $G_{j 0}$ & $G_{i 0}$ & $F_{e}$ & $F_{1}$ & $F_{2}$ & $F_{3}$ & $F_{4}$ & $F_{5}$ & $F_{6}$ & $F_{7}$ & $F_{8}$ & $F_{9}$ & $F_{10}$ & $F_{11}$ & $F_{12}$ \\
\hline$f_{1}$ & 0 & 0 & 1 & $e$ & 1 & 1 & 0 & 0 & 0 & 1 & 0 & 0 & 0 & $e$ & 0 \\
$f_{2}$ & $e$ & $e$ & 1 & $e$ & $e$ & $e$ & $e$ & $e$ & $e$ & $e$ & $e$ & $e$ & $e$ & $e$ & $e$ \\
$f_{3}$ & 1 & 1 & 1 & $e$ & 0 & 0 & 0 & 1 & 0 & 0 & 0 & $e$ & 0 & 0 & 0 \\
$f_{4}$ & $e$ & 0 & 1 & $e$ & 1 & $e$ & $e$ & 0 & 0 & $e$ & $e$ & 0 & 0 & $e$ & $e$ \\
$f_{5}$ & 1 & 0 & 1 & $e$ & 1 & 0 & 1 & 0 & 0 & 0 & $e$ & 0 & 0 & 0 & 0 \\
$f_{6}$ & 0 & $e$ & 1 & $e$ & $e$ & 1 & 0 & 0 & $e$ & $e$ & 0 & 0 & $e$ & $e$ & 0 \\
$f_{7}$ & 1 & $e$ & 1 & $e$ & $e$ & 0 & $e$ & $e$ & 0 & 0 & $e$ & $e$ & 0 & 0 & 0 \\
$f_{8}$ & 0 & 1 & 1 & $e$ & 0 & 1 & 0 & 0 & 1 & 0 & 0 & 0 & $e$ & 0 & 0 \\
$f_{9}$ & $e$ & 1 & 1 & $e$ & 0 & $e$ & 0 & $e$ & $e$ & 0 & 0 & $e$ & $e$ & 0 & 0 \\
\hline
\end{tabular}



Figure 2. The poset of join irreducible elements of the free ternary algebra on two free generators. 
Then,

$$
\begin{aligned}
I P_{1}(G)= & \left\{\left\{G_{i_{0}}\right\},\left\{G_{j_{0}}\right\},\left\{F_{2}\right\},\left\{F_{3}\right\},\left\{G_{i_{0}}, G_{j_{0}}\right\},\left\{F_{2}, F_{3}\right\},\left\{G_{i_{0}}, F_{3}\right\},\left\{F_{2}, G_{j_{0}}\right\}, \varnothing\right\}, \\
I P_{2}(G)= & \left\{\left\{G_{i_{0}}, G_{j_{0}}, F_{e}\right\},\left\{F_{e}, F_{2}, F_{3}\right\},\left\{F_{e}, G_{i_{0}}, F_{3}\right\},\left\{F_{e}, G_{j_{0}}, F_{2}\right\},\left\{G_{i_{0}}, G_{j_{0}}, F_{e}, F_{2}\right\},\right. \\
& \left.\left\{G_{i_{0}}, G_{j_{0}}, F_{e}, F_{3}\right\},\left\{F_{e}, G_{i_{0}}, F_{2}, F_{3}\right\},\left\{F_{e}, G_{j_{0}}, F_{2}, F_{3}\right\},\left\{F_{e}, G_{i_{0}}, G_{j_{0}}, F_{2}, F_{3}\right\}\right\} .
\end{aligned}
$$

The poset IP coincides with that obtained by R. Balbes in [7] and it is the one indicated in Figure 2.

\section{References}

[1] Brzozowski, J.A. and Serger, C.J. (1995) Asynchronous Circuits. Springer-Verlag, Berlin. http://dx.doi.org/10.1007/978-1-4612-4210-9

[2] Sankappanavar, H.P. (1980) A Characterization of Principal Congruences of De Morgan Algebras and Its Applications. In: Arruda, A.I., Chuaqui, R. and da Costa, N.C.A., Eds., Mathematical Logic in Latin American, North-Holland Publishing Company, Amsterdam, 341-349.

[3] Day, A. (1971) A Note on the Congruence Extension Property. Algebra Universalis, 1, 234-235. http://dx.doi.org/10.1007/BF02944983

[4] Blok, W.J. and Pigozzi, D. (1982) On the Structure of Varietes with Equationally Definable Principal Congruences I. Algebra Universalis, 15, 195-227. http://dx.doi.org/10.1007/BF02483723

[5] Kholer, P. and Pigozzi, D. (1980) Varietes with Equationally Definable Principal Congruences. Algebra Universalis, 11, 213-219. http://dx.doi.org/10.1007/BF02483100

[6] Dilworth, R.P. (1945) Lattices with Unique Complements. Transactions of the American Mathematical Society, 57, 123-154. http://dx.doi.org/10.1090/S0002-9947-1945-0012263-6

[7] Balbes, R. (2000) Free Ternary Algebras. International Journal of Algebra and Computation, 10, 739-749. http://dx.doi.org/10.1142/S0218196700000340

[8] Figallo Jr., A. and Ziliani, A. (2011) Free Algebras over a Poset in Varieties. Communications of the Korean Mathematical Society, 26, 543-549. 
Scientific Research Publishing (SCIRP) is one of the largest Open Access journal publishers. It is currently publishing more than 200 open access, online, peer-reviewed journals covering a wide range of academic disciplines. SCIRP serves the worldwide academic communities and contributes to the progress and application of science with its publication.

Other selected journals from SCIRP are listed as below. Submit your manuscript to us via either submit@scirp.org or Online Submission Portal.
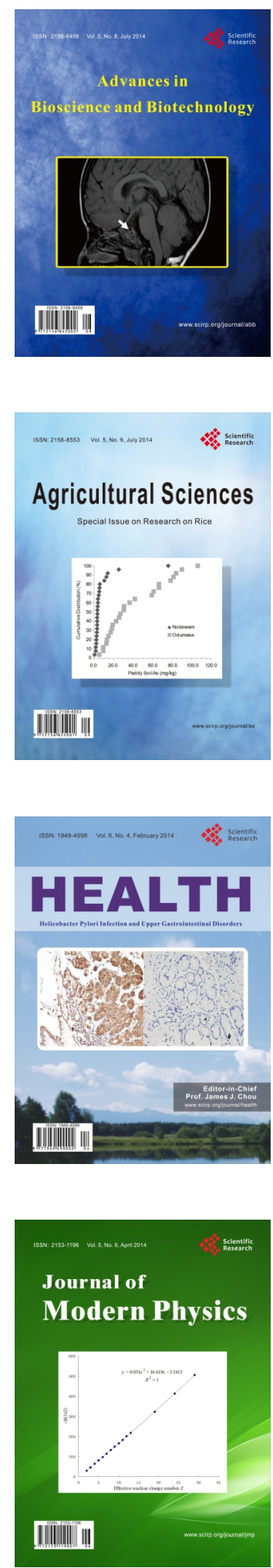
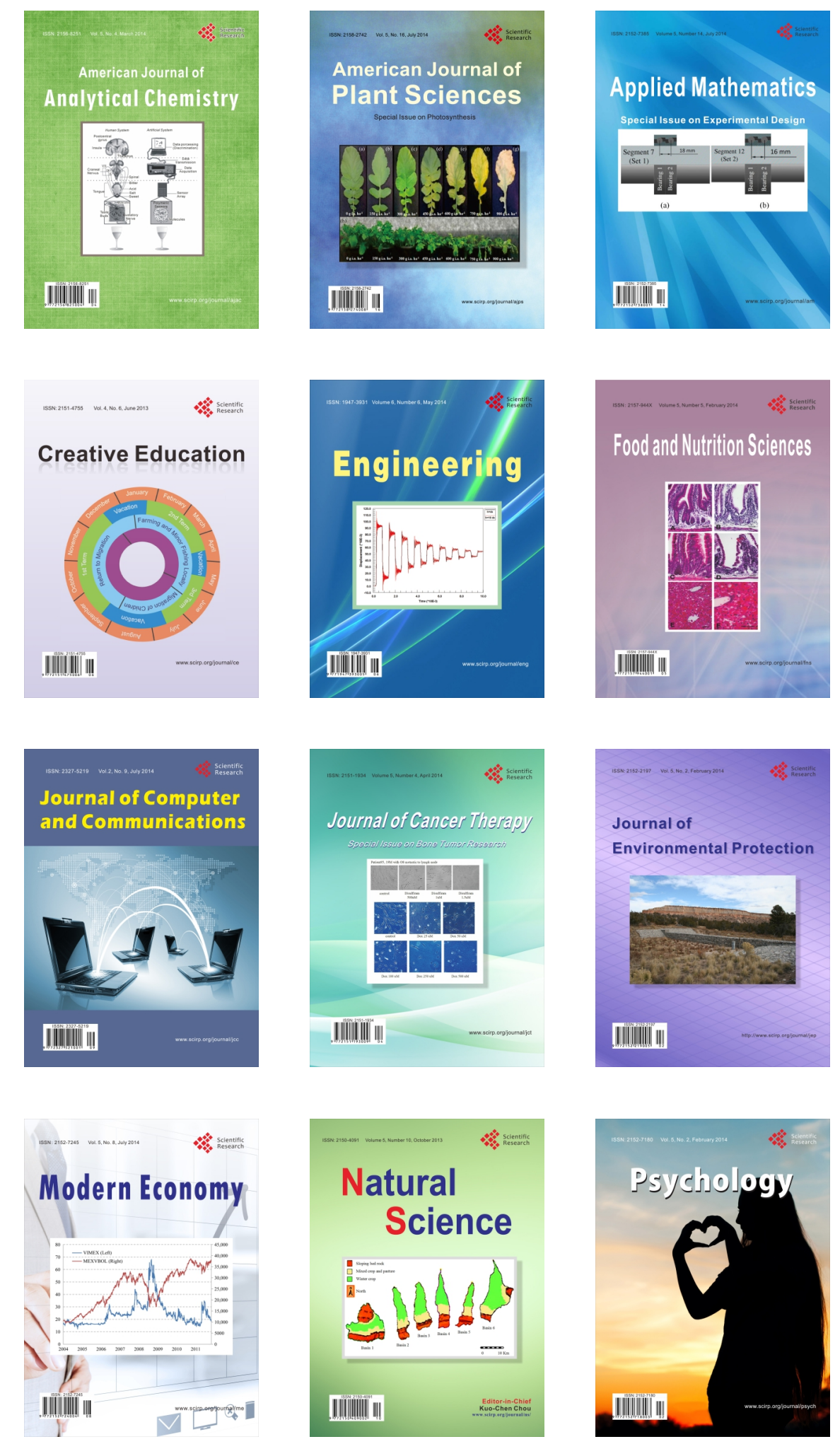